NASA Technical Memorandum 101998

\title{
Numerical Solution of Periodic Vortical Flows About a Thin Airfoil
}

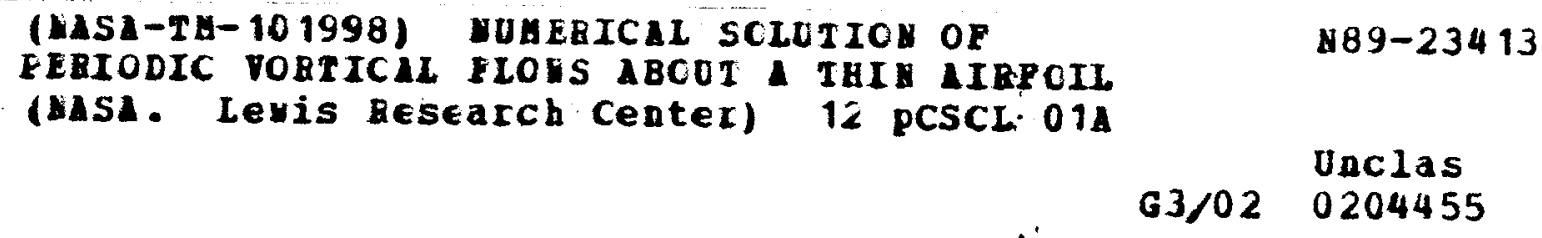

James R. Scott

Lewis Research Center

Cleveland, Ohio

and

Hafiz M. Atassi

University of Notre Dame

Notre Dame, Indiana

Prepared for the

24th Thermophysics Conference

sponsored by the American Institute of Aeronautics and Astronautics Buffalo, New York, June 12-14, 1989 


\title{
NUMERICAL SOLUTION OF PERIODIC VORTICAL FLOWS ABOUT A THIN AIRFOIL
}

\author{
James R. Scott * \\ Lewis Research Center \\ Cleveland, Ohio 44135 \\ and \\ Hafiz M. Atassi ** \\ University of Notre Dame
}

National Aeronautics and Space Administration

Notre Dame, IN 46556

\begin{abstract}
A numerical method is developed for computing periodic, three-dimensional, vortical flows around isolated airfoils. The unsteady velocity is split into a vortical component which is a known function of the upstream flow conditions and the Lagrangian coordinates of the mean flow, and an irrotational field whose potential satisfies a nonconstantcoefficient, inhomogeneous, convective wave equation. Solutions for thin airfoils at zero degrees incidence to the mean flow are presented in this paper. Using an elliptic coordinate transformation, the computational domain is transformed into a rectangle. The Sommerfeld radiation condition is applied to the unsteady pressure on the grid line corresponding to the far field boundary. The results are compared with a Possio solver, and it is shown that for maximum accuracy the grid should depend on both the Mach number and reduced frequency. Finally, in order to assess the range of validity of the classical thin airfoil approximation, results for airfoils with zero thickness are compared with results for airfoils with small thickness.
\end{abstract}

\section{Introduction}

Turbomachinery and propeller blades usually operate in a flow environment where upstream nonuniformities are convected downstream and induce unsteady forces which cause forced vibrations and radiate noise into the far field. Among the sources of such upstream vortical disturbances are atmospheric turbulence, installation effects, inlet distortion, and viscous wakes. For rotating blades, the unsteady disturbances will be periodic in time with a fundamental frequency equal to the blade passing frequency, and will appear in the blade frame of reference as propagating vorticity waves that are called gusts.

The attempt to analyze such unsteady vortical flow fields goes back to the early days of powered flight when engineers sought to deal with vibration problems that arose due to higher flight speeds. For simplicity, investigators

- Research Scientist; Member, AIAA.

- Professor, Aerospace and Mechanical Engineering; Associate Fellow, AIAA. at that time considered flat plate airfoils ab zero mean incidence with small amplitude, harmonic velocity disturbances imposed upstream. In this case the underlying mean flow is a uniform parallel flow, and the vorticity waves are convected by the mean flow without distortion. The standard mathematical treatment consisted of splitting the unsteady velocity field into solenoidal and irrotational (potential) components. The solenoidal component is a known function of the upstream disturbance and represents a purely convected vorticity wave which is completely decoupled from the steady state aerodynamics. The unsteady potential is coupled to the vortical component only through the airfoil boundary condition, and satisfies a constant coefficient, homogeneous, convective wave equation, which for incompressible flows reduces to Laplace's equation. The analyt ical tools used by researchers at that time were primarily the simple concepts of circulation theory and complex variable techniques. Sears ${ }^{1}$ derived an analytical solution for the unsteady lift on a rigid airfoil passing through a vortical sinusoidal gust in incompressible flow. When the flow is compressible, the mathematical problem can be formulated in the form of an integral equation known as Possio's equation ${ }^{2}$. Numerical results are usually obtained by collocation techniques.

For flows around real airfoils with thickness, camber, or angle of attack, the mean flow is no longer a uniform parallel flow. Goldstein and Atassi ${ }^{3}$ showed that in this case the oncoming vortical waves (gusts) are distorted as they are convected by the mean flow. The distortion causes a significant variation in both the amplitude and phase of the unsteady velocity field associated with the gust. Goldstein and Atassi ${ }^{3}$ and Atassi ${ }^{4}$ derived explicit analytical formulas for the unsteady lift of an airfoil subject to a longitudinal and transverse gust in incompressible flow. Their results show that the gust distortion has a significant effect on the airfoil unsteady lift.

For subsonic and transonic flows about lifting airfoils, the steady mean flow is usually obtained numerically. The more complex mathematical problem of unsteady flows also requires numerical computations. There are two basic directions that a computational approach may take. The first of these is the so-called primitive variable approach wherein a system of governing partial differential equations, such as 
the Euler or Navier-Stokes equations, are solved along with certain specified boundary conditions. In recent years a considerable amount of work has been done in this area and much progress has been made. This approach has been used by a number of investigators to calculate numerical solutions to complex unsteady, vortical flow problems such as rotor-stator or wake-rotor interaction. References 5 through 8 represent some of the recent work in this area. The main disadvantage of the primitive variable approach is that a complete system of unsteady, nonlinear partial differential equations must be solved. Large computer memory and lengthy computational times are normally required, and uncertainties about far field boundary conditions for time dependent calculations governed by nonlinear equations leave some question about the accuracy of the solutions. More progress needs to be made in the areas of efficient, accurate algorithms and accurate far field boundary conditions for nonlinear problems to make the primitive variable approach more generally useful.

The alternative to the primitive variable approach is a linearized approach which makes use of the so-called "rapid distortion" approximation. For mean potential flows with small amplitude vortical and entropic disturbances imposed upstream, the unsteady velocity field can be split into the sum of a known vortical component and an unknown potential component that satisfies a linear, nonconstant-coefficient, inhomogeneous, convective wave equation. Details concerning the derivation of this equation are found in references 9 and 10 .

The major limitation of the linear approach is that it is valid for only small amplitude disturbances where the magnitude of the unsteady velocity perturbations is an order of magnitude less than the magnitude of the mean velocity. However, for many pracitcal flows, and in particular many turbomachinery flows, this condition is satisfied so that the limitation is not serious.

On the other hand, the advantages of a linear scheme over a nonlinear approach are significant. Among the advantages are the fact that it is far simpler to derive stable, accurate differencing schemes for a single linear equation than for a nonlinear system of equations, and the computational time required to obtain a solution can be up to an order of magnitude less. In addition, it is easier to derive far field boundary conditions that are accurate and correctly model the physics of the unsteady flow. Since incorrect or inaccurate far field boundary conditions can lead to large errors in the solution of an unsteady calculation, this is indeed a major advantage.

The authors are in the process of implementing a general numerical scheme which can solve arbitrary, small amplitude, periodic vortical flows about isolated airfoils with complex geometry for a large range of Mach numbers and reduced frequencies. In a previous paper ${ }^{11}$, we presented the linear aeordynamic theory for such flows, and showed that the most general upstream vortical disturbances can be represented as the sum of three-dimensional vorticity waves in the blade frame of reference. Numerical results were presented for the special case of thick, symmetric airfoils with imposed upstream three-dimensional, harmonic velocity disturbances. However, as our major purpose in [11] was to present the formulation of the problem, we did not provide the details of our numerical scheme. In the present paper we present the details of the numerical scheme that has been developed. For simplicity, and also to assess the accuracy of the scheme, we concentrate on the thin airfoil problem for which there are known solutions with which comparison can be made. The differences between the general case of distorted vortical flows versus the classical thin airfoil case where there is no distortion will also be pointed out. In addition, we discuss specific numerical difficulties such as the choice of appropriate grids and coordinate transformation, far field boundary condition, and linear solution technique. In section II we present the mathematical formulation of the boundary value problem for the general case and show the simplifications that result from the thin airfoil approximation. In section III we describe our numerical scheme, and in Section IV we present comparisons between computed results and known solutions to the classical problems. We also present comparisons between results for flat plate airfoils and airfoils with small thickness in order to assess the range of validity of the thin airfoil approximation.

\section{Mathematical Formulation of the Boundary Value Problem}

\section{Governing Equation}

Consider a two-dimensional airfoil with chord length $\mathrm{c}$ placed at nonzero incidence in a flow with uniform upstream velocity $U_{\infty}$ in the $x_{1}$ direction. Far upstream, let $\vec{u}_{\infty}$ be a small amplitude, sinusoidal gust of the form $\vec{a} e^{i \vec{k} \cdot\left(\vec{r}-i U_{\infty} t\right)}$ which is imposed on the flow (See Figure 1). Here $\vec{a}=$ $\left(a_{1}, a_{2}, a_{3}\right)$, where the amplitude $|\vec{a}|$ satisfies $|\vec{a}|<<U_{\infty}$, $\vec{k}=\left(k_{1}, k_{2}, k_{3}\right)$ is the wave number vector which specifies the direction of propagation of the gust, and $\vec{i}$ is a unit vector in the $x_{1}$ direction. We also assume the fluid to be an ideal gas which is inviscid and non-heat conducting, and require $\vec{a} \cdot \vec{k}=0$ to satisfy the continuity equation. Since we consider periodic disturbances, $\vec{u}_{\infty}$ can be considered as a single Fourier component of the upstream disturbance.

We assume that the velocity field can be represented by

$$
\vec{U}(\vec{x}, t)=\vec{U}_{0}(\vec{x})+\vec{u}(\vec{x}, t)
$$

where $\vec{U}_{0}(\vec{x})$ is the mean potential flow and $\vec{u}(\vec{x}, t)$ represents the small, unsteady part of the velocity field. It is shown in [9] and [10] that $\vec{u}(\vec{x}, t)$ may be decomposed into the sum of a known vortical component $\vec{u}^{(R)}$, and an unknown potential component $\vec{\nabla} \phi$, so that

$$
\vec{u}(\vec{x}, t)=\vec{u}^{(R)}+\vec{\nabla} \phi .
$$

The vortical component $\vec{u}^{(R)}$ is essentially a function of the mean flow Lagrangian coordinates and their spatial gradients. In the case of thin, uncambered airfoils at zero degrees incidence, however, the unsteady velocity decouples from the mean flow and $\vec{u}^{(R)}$ reduces to $\vec{u}_{\infty}$. 
The potential $\phi$ satisfies the convective wave equation

$$
\frac{D_{0}}{D t}\left(\frac{1}{c_{0}{ }^{2}} \frac{D_{0} \phi}{D t}\right)-\frac{1}{\rho_{0}} \vec{\nabla} \cdot\left(\rho_{0} \vec{\nabla} \phi\right)=\frac{1}{\rho_{0}} \vec{\nabla} \cdot\left(\rho_{0} \vec{u}^{(R)}\right)
$$

where $\frac{D_{0}}{D t}$ is the convective derivative associated with the mean flow, and $c_{0}$ and $\rho_{0}$ are, respectively, the mean flow speed of sound and density. The unsteady perturbation pressure is given by

$$
p=-\rho_{0}(\vec{x}) \frac{D_{0} \phi}{D t}
$$

In the general case where the mean flow is nonuniform, the coefficients of the equation and its source term depend strongly on the mean flow quantities. However, for thin airfoils in a uniform parallel flow, the coefficients become constant and the source term vanishes. In this case the equation reduces to

$$
\frac{1}{c_{0}{ }^{2}} \frac{D_{0}^{2} \phi}{D t^{2}}-\nabla^{2} \phi=0
$$

which is the convective wave equation for thin, unloaded airfoils in a uniform mean flow. Note that in the incompressible case the equation reduces to Laplace's equation.

\section{Boundary Conditions}

At the airfoil surface the boundary condition is the usual requirement that the normal velocity component vanish. For the general case this leads to the requirement that $\left(\vec{u}^{(R)}+\vec{\nabla} \phi\right) \cdot \vec{n}=0$ or $\frac{\partial \phi}{\partial n}=-\vec{u}^{(R)} \cdot \vec{n}$. For a flat plate airfoil at zero degress angle of attack this simplifies to

$$
\begin{gathered}
\frac{\partial \phi}{\partial x_{2}}=-a_{2} e^{i \vec{k} \cdot\left(\vec{x}-\vec{i} U_{\infty} t\right)} \\
\text { or } \\
\frac{\partial \phi}{\partial x_{2}}=-a_{2} e^{i k_{1}\left(x_{1}-U_{\infty} t\right)+i k_{3} x_{3}}
\end{gathered}
$$

for $-\frac{c}{2} \leq x_{1} \leq \frac{c}{2}$, and $x_{2}=0$.

In the wake the pressure is continuous, but $\phi$ has a discontinuity $\Delta \phi$ due to the unsteady circulation about the airfoil. Applying (2.4) on each side of the vortex sheet behind the airfoil leads to

$$
\frac{D_{0}}{D t}(\Delta \phi)=0
$$

for $x_{1}>\frac{c}{2}, x_{2}=0$.

Finally, in the far field $\phi$ should satisfy

$$
\vec{\nabla} \phi \rightarrow 0 \text { as } x_{1} \rightarrow-\infty
$$

With the condition that $\phi$ is continuous at the trailing edge, equation (2.5) with conditions (2.7) - (2.9) completely defines the mathematical formulation for the aerodynamic response of a thin airfoil to a sinusoidal gust. For a more detailed discussion of the mathematical formulation of the gust response problem, the reader may consult reference (12).

\section{Numerical Scheme}

\section{Frequency Domain Approach}

In what follows we assume that all lengths have been normalized by $\frac{c}{2}$, time by $\frac{c}{2 U_{\infty}}, \phi$ by $\frac{c}{2}|\vec{a}|, \vec{a}$ by $|\vec{a}|$, and $U_{\infty}$ and $c_{0}$ by $U_{\infty}$. With this nondimensionalization, equation 2.5 and boundary conditions $2.7-2.9$ become

$$
\left.\begin{array}{c}
M^{2} \frac{D^{2} \phi}{D t^{2}}-\nabla^{2} \phi=0 \\
\frac{\partial \phi}{\partial x_{2}}=-a_{2} e^{i k_{1}\left(x_{1}-t\right)+i k_{3} x_{3}} \\
\text { for }-1 \leq x_{1} \leq 1, \quad x_{2}=0
\end{array}\right\}
$$

where $\frac{D}{D t}=\frac{\partial}{\partial t}+\frac{\partial}{\partial x_{1}}$. The normalized wave number $k_{1}=$ $\frac{\nu c}{2 U_{\infty}}$, where $\nu$ is the angular frequency of the upstream disturbance, is commonly referred to as the reduced frequency.

In attempting to solve the boundary value problem (3.1) - (3.4) numerically, there are two basic directions that one can take - the time domain approach or the frequency domain approach. The authors have chosen to work in the frequency domain and so our discussion will be limited to that particular approach. It should be pointed out that for mathematical convenience the classical work on the thin airfoil unsteady flow problems was also carried out in the frequency domain. By using this approach along with a suitable coordinate transformation, equation (3.1) can be reduced to a Helmholtz equation and thus considerable mathematical simplification is obtained.

In order to transform into the frequency domain and reduce equation (3.1) to a Helmholtz equation the following transformation of coordinates is introduced.

$$
\begin{gathered}
x=x_{1}, \quad y=\beta x_{2}, \quad z=x_{3} \text { where } \beta^{2}=1-M^{2} \\
\text { and } \\
\varphi=\phi e^{i \frac{k_{1} M^{2}}{\rho^{2}} x} e^{i k_{1} t-i k_{3} z}
\end{gathered}
$$

With this change of variables equation (3.1) reduces to

$$
\frac{\partial^{2} \varphi}{\partial x^{2}}+\frac{\partial^{2} \varphi}{\partial y^{2}}+\left[\left(\frac{k_{1} M}{\beta^{2}}\right)^{2}-\left(\frac{k_{3}}{\beta}\right)^{2}\right] \varphi=0
$$

which is a Helmholtz equation in the transformed "Prandtl Glauert" plane. Boundary conditions (3.2) - (3.4) have also been modified so that they become

$$
\begin{gathered}
\frac{\partial \varphi}{\partial y}=-\frac{a_{2}}{\beta} e^{i k_{1} x} \quad \text { for } \quad-1 \leq x \leq 1, \quad y=0 \\
\left(-\frac{i k_{1}}{\beta^{2}}+\frac{\partial}{\partial x}\right)(\Delta \varphi)=0 \text { for } x>1, \quad y=0 \\
\vec{\nabla} \varphi \rightarrow 0 \text { as } x \rightarrow-\infty
\end{gathered}
$$

Equation (3.6) together with boundary conditions (3.7) (3.9) represent the frequency domain formulation of the boundary value problem (3.1) - (3.4). 


\section{Numerical Method}

While equation (3.6) represents a significant mathematical simplification over equation (3.1), the independent variables $(x, y)$ of the Prandtl Glauert plane are not ideally suited coordinates for numerical computations. A transformation of the independent variables is needed which will provide adequate resolution of the flow field near the airfoil and also provide a distribution of grid points in the far field which is suitable for acoustic wave propagation and the implementation of far field boundary conditions. To satisfy these requirements, we introduce elliptic coordinates $(\eta, \xi)$ with the transformation

$$
\begin{aligned}
& x=\cos (\pi \eta) \cosh (\pi \xi) \\
& y=\sin (\pi \eta) \sinh (\pi \xi) .
\end{aligned}
$$

Equation (3.6) becomes

$$
\left.\begin{array}{c}
\frac{\partial^{2} \varphi}{\partial \eta^{2}}+\frac{\partial^{2} \varphi}{\partial \xi^{2}}+ \\
\pi^{2}\left[\left(\frac{k_{1} M}{\beta^{2}}\right)^{2}-\left(\frac{k_{3}}{\beta}\right)^{2}\right]\left[\sin ^{2}(\pi \eta)+\sinh ^{2}(\pi \xi)\right] \varphi=0
\end{array}\right\}
$$

and boundary conditions (3.7) - (3.9) are modified accordingly.

In order to obtain numerical solutions to equation (3.11) and its associated boundary conditions, we discretize the computational domain and employ finite differences to represent the governing equation and boundary conditions. The governing equation itself is modelled using the standard, five point central differencing scheme which is second order accurate in both $\eta$ and $\xi$.

The airfoil boundary condition (3.7) has been transformed under the change of variables (3.10) to

$$
\begin{gathered}
\frac{\partial \varphi}{\partial \xi}=-\frac{\pi a_{2}}{\beta} \sin (\pi \eta) e^{i \frac{k_{1} \cos (\pi \eta)}{\beta^{2}}} \\
\text { for } \quad 0<\eta<1, \quad \xi=0,
\end{gathered}
$$

and is represented by four-point, third order accurate, onesided differencing.

It should be pointed out that due to the symmetry of the boundary value problem (3.1) - (3.4) it is necessary to solve (3.11) and its associated boundary conditions only in the upper half plane. The function $\varphi$ is an odd function with respect to $y$, and so we impose the condition $\varphi=0$ on the strcamline ahead of the airfoil. This corresponds to the line $\eta=1, \xi \geq 0$ in the computational plane. At the trailing edge point, we impose the Kutta condition, i.e., the unsteady velocity is finite. Since the Jacobian of transformation (3.10) vanishes at this point, this requires that $\frac{\partial \varphi}{\partial \eta}=0$ at $\eta=0, \xi=0$. This condition is implemented using four-point, one-sided differencing.

In the wake behind the airfoil, $\varphi$ is not continuous but must satisfy a jump condition determined by the continuity of the unsteady pressure. By integrating equation (3.8), one obtains

$$
\Delta \varphi=\Delta \varphi_{\text {t.e. }} e^{i \frac{k_{1}}{\beta^{2}}(x-1)}, \quad x>1, \quad y=0,
$$

where the subscript t.e. denotes quantities evaluated at the trailing edge. $\Delta \varphi_{\text {t.e. }}$ is determined by Kelvin's theorem of conservation of the total circulation in the flow. This is satisfied by assuming that $k_{1}$ has a small positive imaginary part to ensure convergence at infinity. Since $\varphi$ is an odd function of $y, \varphi=\frac{1}{2} \Delta \varphi$ for $y>0$. Expressing this result in terms of $\eta$ and $\xi$ one obtains (3.14).

$$
\varphi=\varphi_{\text {i.e. }} e^{i \frac{\xi_{1}}{\rho^{2}}[\cosh (\pi \xi)-1]} \quad \eta=0, \quad \xi>0
$$

To complete the formulation of the boundary value problem, it is only necessary to impose a condition for grid points on the far field boundary. While condition (3.9) expresses the mathematical requirement that $\vec{\nabla} \varphi \rightarrow 0$ at infinity, applying this condition on a boundary at a finite distance in the computational domain would impose a reflecting boundary condition which will not allow outgoing acoustic waves to leave the computational domain. The reflected acoustic waves will propagate inwards and may cause cause large errors in the solution. It is essential, therefore, to have a radiation type boundary condition which will allow outgoing waves to leave the solution domain. Moreover, the radiation condition should be applied to a continuous flow variable such as the pressure. The authors have implemented a variety of radiation conditions including a series expansion for $\varphi$, a Sommerfeld radiation condition on $\varphi$, and a Sommerfeld radiation condition on the pressure $p$. While each approach gave a good near field solution, only the pressure radiation condition gave both a good near field solution and correct far field acoustics.

It can be shown that the Sommerfeld radiation condition applied to the pressure is given in operator notation by

$$
\left[\frac{\partial}{\partial r}-i \sqrt{\left(\frac{k_{1} M}{\beta^{2}}\right)^{2}-\left(\frac{k_{3}}{\beta}\right)^{2}}\right]\left(\frac{\partial}{\partial x}-i \frac{k_{1}}{\beta^{2}}\right) \varphi=0
$$

where $\mathrm{x}=\mathrm{r} \cos \theta, \mathrm{y}=\mathrm{r} \sin \theta$. Neglecting $\frac{1}{r} \frac{\partial}{\partial \theta}$ terms, this reduces to

$$
\left.\begin{array}{c}
\cos \theta \frac{\partial^{2} \varphi}{\partial r^{2}}-i\left[\sqrt{\left(\frac{k_{1} M}{\beta^{2}}\right)^{2}-\left(\frac{k_{3}}{\beta}\right)^{2}} \cos \theta+\frac{k_{1}}{\beta^{2}}\right] \frac{\partial \varphi}{\partial r} \\
-\left[\frac{k_{1}}{\beta^{2}} \sqrt{\left(\frac{k_{1} M}{\beta^{2}}\right)^{2}-\left(\frac{k_{3}}{\beta}\right)^{2}}\right] \varphi=0
\end{array}\right\}
$$

This condition is applied for all grid points such that $0<$ $\eta<1, \xi=\xi_{\max }$, and is implemented using four point, one-sided differencing.

\section{Solution Technique and Grid Selection}

Obtaining the solution to the finite difference equations representing equation (3.11) and its associated boundary conditions requires solving a large matrix equation for the unkown solution values $\varphi_{i, j}$. While the matrix has a block 
structure, it is not block tridiagonal due to the wake boundary condition (3.14), so that standard block solvers cannot be used. Experience has also shown that standard iterative solvers have convergence problems due to the fact that the governing equation is a Helmholtz equation with Mach number and wave number dependency. In light of these difficulties, a direct, general purpose, sparse matrix solver was developed which stores only the nonzero entries of the matrix, and performs the elimination with or without pivoting. The typical number of equations to be solved varied from 2,000 to 4,000, with solution times ranging from 15 to 200 seconds on a Cray X/MP, depending on the Mach number and reduced frequency. Pivoting during the elimination has peen found to be unnecessary.

The final issue to be discussed in regard to our numerical scheme is the method of grid selection. Because the gust problem is essentially a wave propagation problem, it is necessary to ensure that for any given calculation the grid spacing is such that the waves can be accurately resolved. This requires having sufficient spacing to resolve both the airfoil boundary condition (3.12) and the wake boundary condition (3.14). Experience has shown that the spacing in the $\xi$ direction should be uniform near the airfoil but in the far field should be chosen so that the argument of the exponential function in (3.14) should vary by equal fractional increments of $\pi$, say $\frac{\pi}{6}$, for example, for maximum accuracy. This requires the $\xi$ spacing to depend on both $k_{1}$ and $M$. In addition, the accuracy of the far field boundary condition (3.16) depends upon the reduced frequency, the Mach number, and the distance $R$ to the far field boundary in such a way that the product $\frac{k_{1} M}{\beta^{2}} R$ should remain large. This requires the location of the outer boundary to be a function of $k_{1}$ and $\mathrm{M}$. We conclude on the basis of these observations that for accurate calculation of a complete range of $\mathrm{Mach}$ numbers and reduced frequencies in the frequency domain formulation, the unsteady grid must be determined as a function of both Mach number and reduced frequency.

\section{Results And Discussion}

\section{Analysis of Results}

To validate our numerical scheme we calculate solutions to the classical thin airfoil vortical flow problems and compare the numerical results with known solutions. For purposes of this comparison we define the unsteady response function

$$
R\left(k_{1}, k_{3}, M\right)=\frac{L^{\prime}}{\pi \rho c U_{\infty}|\vec{a}| e^{i \nu t}}
$$

where $L^{\prime}$ is the unsteady lift. Comparison will be made for flows with Mach numbers of $.1, .5$, and .8 , and with reduced frequencies that range from 0 to 4.01. Sample grids used during the calculation are shown in Figures 2 through 5.

In Figure 6 we compare the computed unsteady response function with the Sears solution for the case of a flat plate airfoil in a transverse gust $\left(a_{2}=1, a_{1}=a_{3}=k_{2}=\right.$ $k_{3}=0$ ) at a Mach number of .1. The reduced frequency values used for this and all other calculations are shown in Table I. The point on the plot which is on the real axis and furthest to the right corresponds to the quasi-steady case in which $k_{1}=0$, and the other $k_{1}$ values correspond in order to the other points along the curve. The agreement between the numerical results and the Sears solution is excellent except for a small discrepancy at the high frequency end.

Figures 7 and 8 show plots for the case of a flat plate airfoil in a transverse gust with a .5 and $.8 \mathrm{Mach}$ number, respectively. Again, the agreement between the numerical and analytical results is quite good. Note also that the effect of the Mach number is to increase the magnitude of the unsteady response for the low frequencies and decrease it for the high frequencies.

As mentioned previously, the grids used for the computations are determined as a function of Mach number and reduced frequency. Figures 9 through 11 show the effect of calculating the results on grids independent of $k_{1}$. For each case shown, the same grid was used for all frequencies in the calculation. The grid used for each Mach number was the one normally used only for the highest frequency case. By using the grid for the highest frequency, it was assured that the grid resolution for the lower frequencies would be more than adequate. But as the results show, the accuracy of the calculation is not nearly as good as when the grid is determined as a function of $k_{1}$. This is the result of keeping the far field boundary fixed, rather than adjusting it with frequency. Note that as the Mach number increases, the agreement becomes progressively worse.

In figures 12 through 14 we compare computed results with results from a Possio solver for three dimensional gusts. The conditions on the gust wave number parameters are shown below the plot. As in the case of the transverse gust, the point on the plot which is on the real axis and furthest to the right corresponds to $k_{1}=0$, and the other $k_{1}$ values correspond in order to the other points along the curve. The agreement is in general very good. The small discrepancy in the results for the .8 Mach number case is due to a change in sign of the term $\left[\left(\frac{k_{1} M}{\beta^{2}}\right)^{2}-\left(\frac{k_{3}}{\beta}\right)^{2}\right]$ which multiplies $\varphi$ in equation (3.11). The sign change occurs at $k_{1}=0.332$. For reduced frequencies near 0.332 the solution to the problem is very sensitive to small changes in $k_{1}$ so that it becomes more difficult to obtain accurate numerical solutions.

To complete our discussion of the the thin airfoil gust response problem, and to assess the range of validity of the thin airfoil approximation, we present comparisons between calculations for flat plate airfoils with zero thickness and Joukowski airfoils with $3 \%$ thickness. For airfoils with nonzero thickness it is necessary to solve the full governing equation (2.3) and boundary conditions modified to take into account the nonuniformities in the mean flow. For details concerning the solution of equation (2.3) for airfoils 
with thickness, the reader should consult [11]. Comparisons are macte for 1-D (transverse), 2-D, and 3-D gusts, and for Mach numbers of .1 and .6. See Figures 15 through 20 .

An analysis of the results indicates that the thickness has neglibible effect when the flow is incompressible, except for the 2-D case for reduced frequencies above about 2.0, where the magnitude of the response has been reduced by about $20 \%$. For the .6 Mach number case, the thickness has a significant effect on the response function for both the 1-D and 2-D cases. For the 1-D case the effect of the thickness is to increase the magnitude of the response by about $2 \%$ at the low frequencies and decrease it by about $10 \%$ at the higher frequencies. For the 2-D case the effect is primarily limited to the higher frequencies and amounts to a decrease of about $15 \%$.

\section{Conclusion}

We conclude on the basis of the excellent agreement in the results that have been presented that the numerical scheme which has been developed can calculate with high accuracy the solution to the thin airfoil, unsteady vortical flow problems for a large range of Mach numbers and reduced frequencies. Moreover, the scheme gives not only a good near field solution but also provides an accurate solution in the far field from which the acoustics can be readily calculated. The key ingredients in the scheme include the transformation into elliptical coordinates, determination of the grid as a function of the Mach number and reduced frequency, implementation of a radiation condition for the pressure in the far field, and the development of a direct, storage efficient sparse matrix solver which can be used for sparse matrices of arbitrary structure.

The extension of our method to more general flows is relatively straightforward. A future paper will discuss the specific details of this extension, and also present numerical results for periodic, vortical flows past airfoils with arbitrary geometry.

Acknowledgement:

The authors would like to thank Dr. John F. Groeneweg for his support throughout this work. The work of one of the authors (H.M.A.) was supported by NASA Lewis Research Center under grant number NAG3732.

\section{REFERENCES}

1. Sears, W. R., "Some Aspects of Non-stationary Airfoil Theory and Its Practical Applications," J. Aero. Sci., 81, 3, 1941, pp. 104-108.

2. Possio, C., "L'Azione Aerodinamica sul Profilo Oscillante in un Fluido Compressible a Velocita Ipsonora." $L$ 'Aerotecnica XVIII, 4, 1938.

3. Goldstein, M. E. and Atassi, H. M., "A Complete Second-Order Theory for the Unsteady Flow About an Airfoil Due to a Periodic Gust," J. Fluid Mech., Vol. 74, pp. 741-765, 1976.

4. Atassi, H. M., "The Sears Problem for a Lifting Airfoil Revisited - New Results," J. Fluid Mech., Vol. 141, pp. 109-122, 1984.

5. Giles, M., "Calculation of Unsteady Wake/Rotor Interactions," AIAA Paper 87-0006, Jan. 1985.

6. Rai, M. M., "Unsteady Three Dimensional NavierStokes Simulations of Turbine Rotor-Stator Interaction," AIAA Paper 87-2058, June 1987.

7. Jorgenson, P. C. E. and Chima, R. V., "An Explicit Runge-Kutta Method for Unsteady Rotor/Stator Interaction," AIAA Paper 88-0049, Jan. 1988.

8. Janus, J. M. and Whitfield, D. L., "A Simple TimeAccurate Turbomachinery Algorithm with Numerical Solutions of an Uneven Blade Count Configuration," AIAA Paper 89-0206, Jan. 1989.

9. Goldstein, M. E., "Unsteady Vortical and Entropic Distortions of Potential Flows Round Arbitrary Obstacles," J. Fluid Mech., Vol. 89, 433-468, 1978.

10. Atassi, H. M. and Grzedzinski, J., "Unsteady Disturbances of Streaming Motions Around Bodies," accepted for publication in J. Fluid Mech.

11. Atassi, H. M. and Scott, J. R., "Analysis of Nonuniform Subsonic Flows About a Row of Moving Blades," Proceedings of the Fourth International Symposium on Unsteady Aerodynamics and Aeroelasticity of Turbomachines and Propellers, Eds. H. E. Gallus and S. Servaty, pp. 39-67, Feb., 1988

12. Atassi, H. M., "Unsteady Vortical Disturbances Around Bodies," Proceedings of the Tenth U. S. Na. tional

Congress of Applied Mechanics, ed. J. P. Lamb, pp. 475-484, June, 1986. 
TABLE I. - REDUCED FREQUENCY VALUES FOR FIGURES 6 TO 20

\begin{tabular}{|c|c|}
\hline $\begin{array}{c}\text { Figures } 6 \text { to } 14, \\
k_{1}\end{array}$ & $\begin{array}{c}\text { Figures } 15 \text { to } 20 \\
k_{1}\end{array}$ \\
\hline 0.00001 & 0.00001 \\
.00686 & .01000 \\
.02744 & .03000 \\
.06176 & .06000 \\
.10988 & .10000 \\
.17186 & .20000 \\
.24780 & .30000 \\
.33781 & .45000 \\
.44205 & .60000 \\
.56070 & .80000 \\
.69396 & 1.00000 \\
.84211 & 1.30000 \\
1.0055 & 1.60000 \\
1.1844 & 2.00000 \\
1.3794 & 2.50000 \\
1.5910 & 3.00000 \\
1.8200 & 3.50000 \\
2.0670 & 4.00000 \\
2.3333 & \\
2.6200 & \\
2.9289 & \\
3.2621 & \\
3.6224 & \\
4.0136 & \\
\hline
\end{tabular}

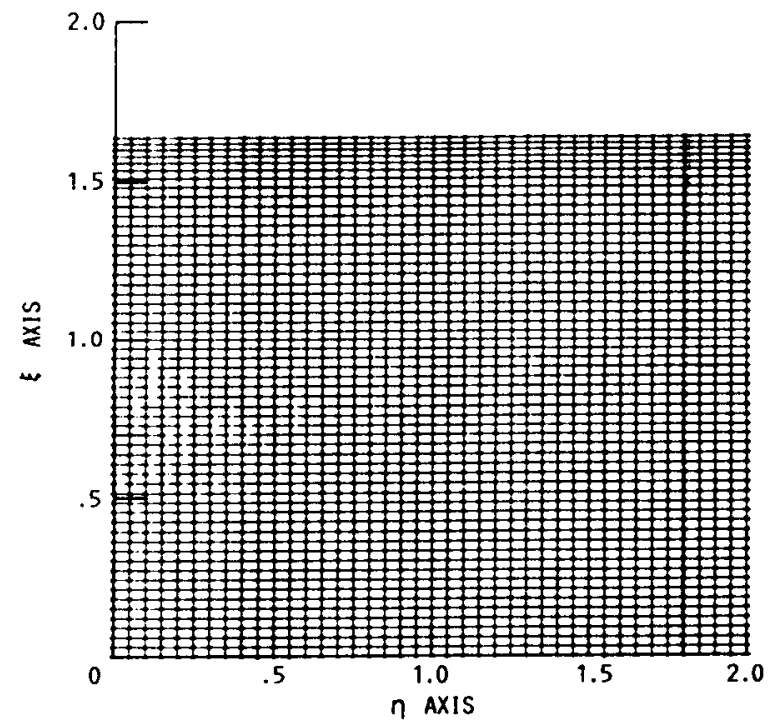

FIGURE 2. - COMPUTATIONAL GRID IN THE TRANSFORHED PLANE FOR $M=.1, k_{1}=.1$.
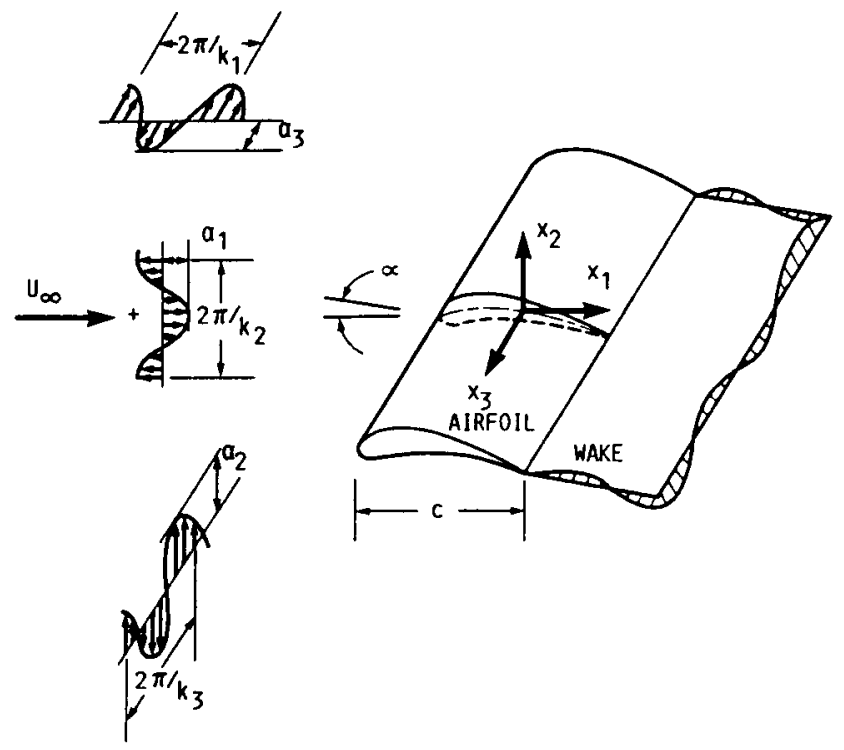

FIGURE 1. - AIRFOIL IN A THREE-DIMENSIONAL GUST.

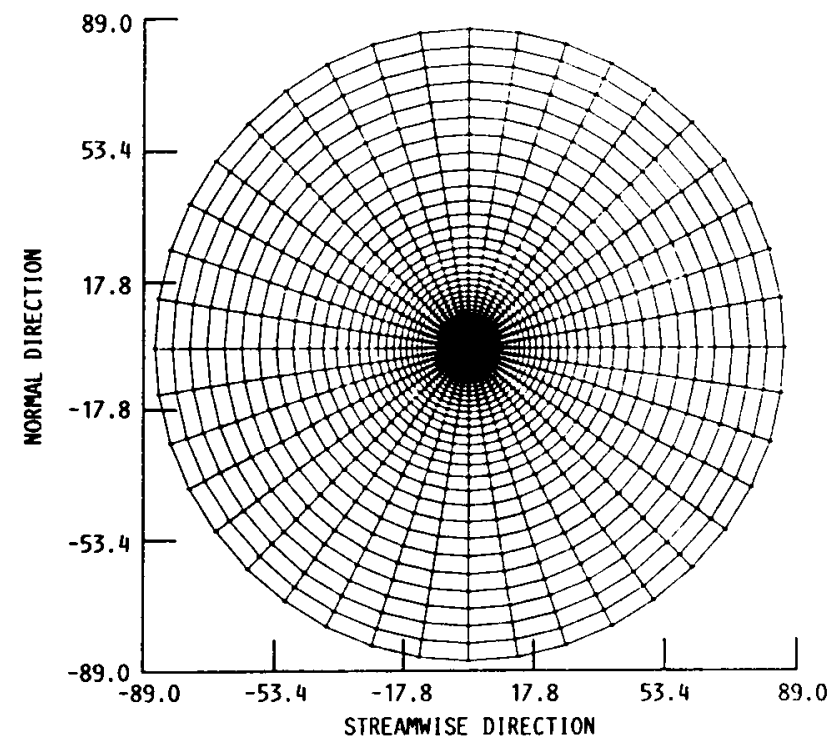

FIGURE 3. - COMPUTATIONAL GRID IN THE PHYSICAL PLANE FOR $M=.1, k_{1}=.1$. 


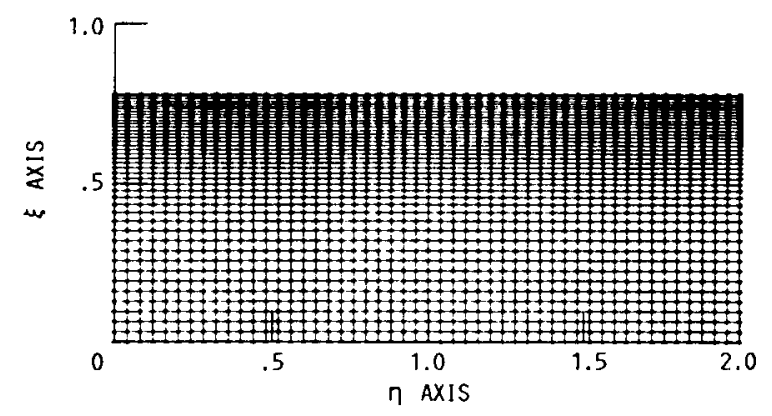

FIGURE 4. - COMPUTATIONAL GRID IN THE TRANSFORMED PLANE FOR $M=.5, k_{1}=3.0$.

UNSTEADY LIFT

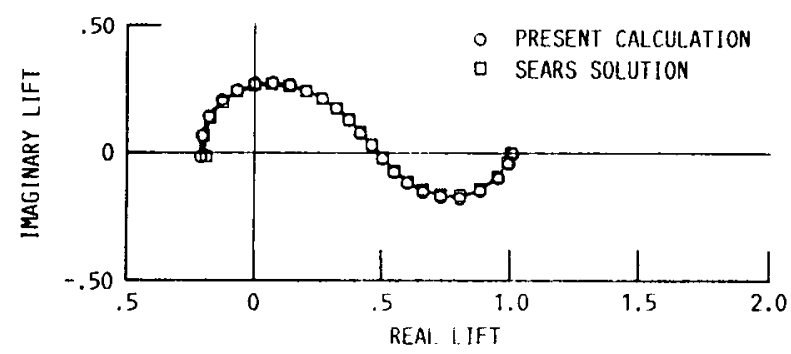

FIGURE 6, - COMPARISON BETWEEN THE COMPUTED UNSTEADY RESPONSE FUNCTION OF A FLAT PIATE IN A TRANSVERSE GUST AT $M=0.1$ AND THE SEARS SOLUTION.

UNSTEADY LIFT

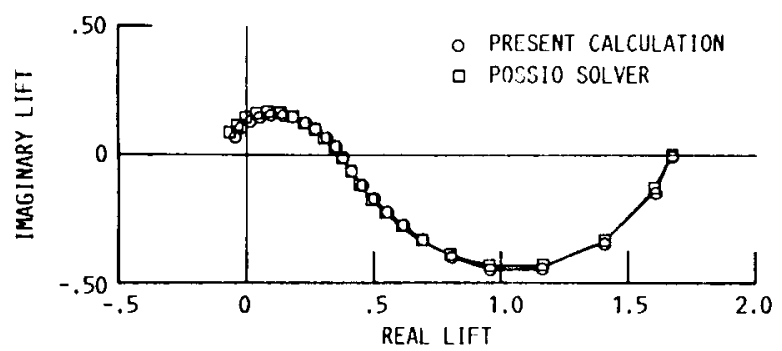

FIGURE 8. - COMPARISON BETWEEN THE COMPUTED UNSTEADY RESPONSE FUNCTION OF A FLAT PL.ATE AIRFOIL IN A TRANSVERSE GUST AT $M=0.8$ AND A POSSIO SOIVER.

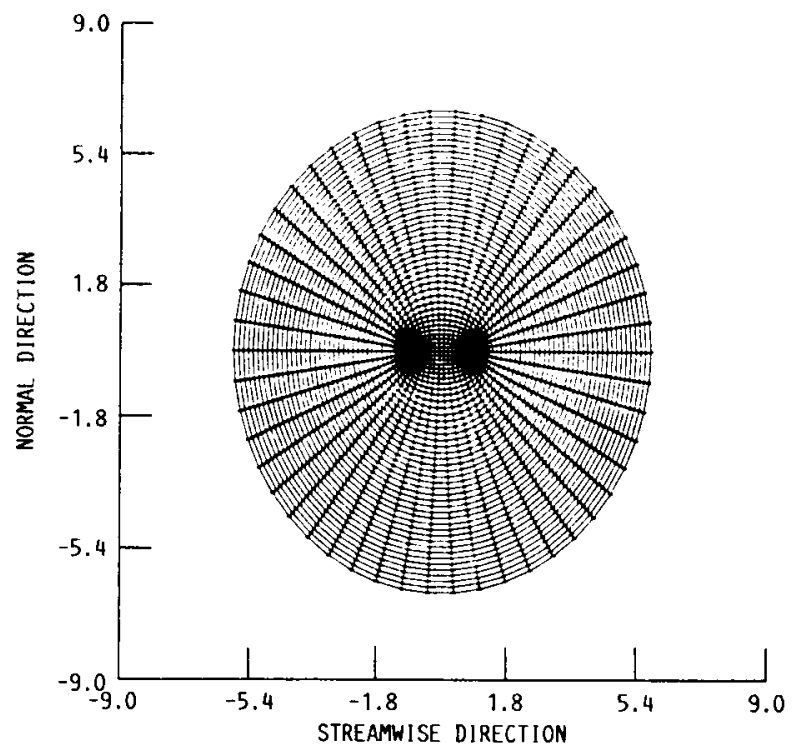

FIGURE 5. - COMPUTATIONAL GRID IN THE PHYSICAL PLANE FOR $M=.5, k_{1}=3.0$.

UNSTEADY LIFT

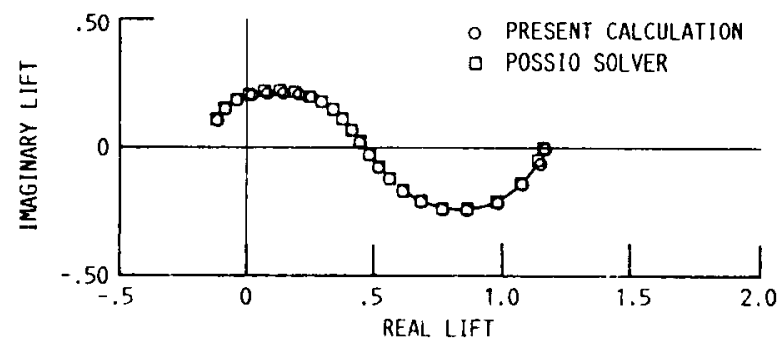

FIGURE 7. - COMPARISON BETWEEN THE COMPUTED UNSTEADY RESPONSE FUNCTION OF A FLAT PLATE AIRFOIL IN A TRANSVERSE GLST AT $M=0.5$ AND A POSSIO SOLVER.

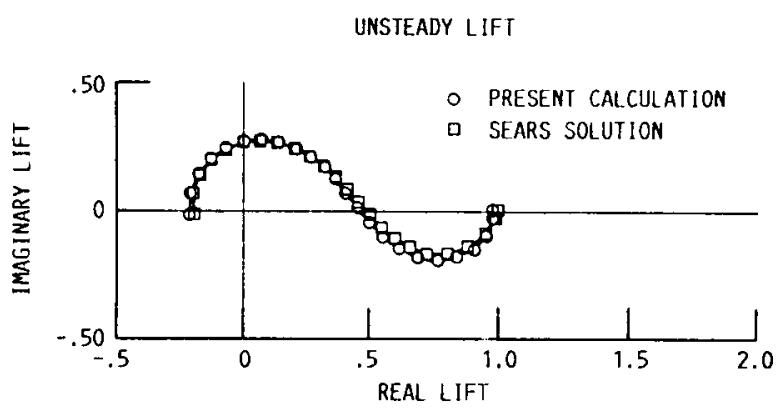

FIGURE 9. - COMPARISON BETWEEN THE COMPUTED UNSTEADY RESPONSE FUNCTION OF A FLAT PLATE AIRFOIL IN A TRANSVERSE GUST AT $M=0.1$ AND THE SEARS SOLUTION. THE UNSTEADY GRID IS THE SAME FOR ALL $k_{1}$. 
UNSTEADY LIFT

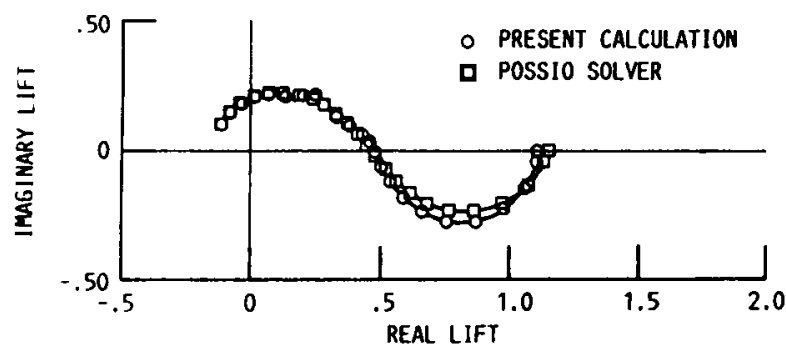

FIGURE 10. - COMPARISON BETWEEN THE COMPUTED UNSTEADY RESPONSE FUNCTION OF A FLAT PLATE AIRFOIL IN A TRANSVERSE GUST AT $M=0.5$ AND A POSSIO SOLVER. THE UNSTEADY GRID IS THE SAME FOR ALL $k_{1}$.

UNSTEADY LIFT

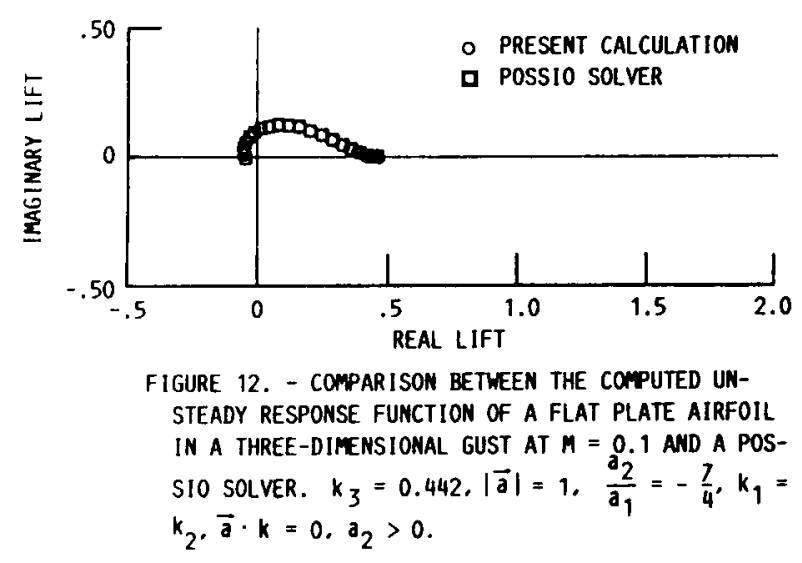

UNSTEADY LIFT

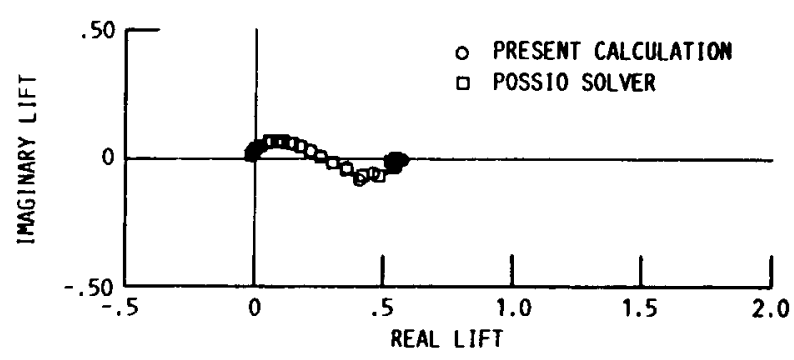

FIGURE 14. - COMPARISON BETWEEN THE COMPUTED UNSTEADY RESPONSE FUNCTION OF A FLAT PLATE AIRFOIL IN A THREE-DIMENSIONAL GUST AT $M=0.8$ AND A POSSIO SOLVER. $\quad k_{3}=0.442,|\vec{a}|=1, \frac{a_{2}}{a_{1}}=-\frac{7}{4}, k_{1}=k_{2}$, $\vec{a} \cdot \vec{k}=0, a_{2}>0$.
UNSTEADY LIFT

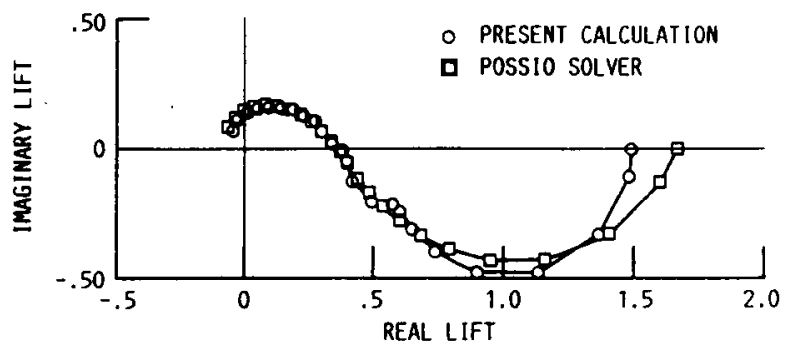

FIGURE 11. - COMPARISON BETWEEN THE COMPUTED UNSTEADY RESPONSE FUNCTION OF A FLAT PLATE AIRFOIL IN A TRANSVERSE GUST AT $M=0.8$ AND A POSSIO SOLVER. THE UNSTEADY GRID IS THE SAME FOR ALL $k_{1}$.

UNSTEADY LIFT

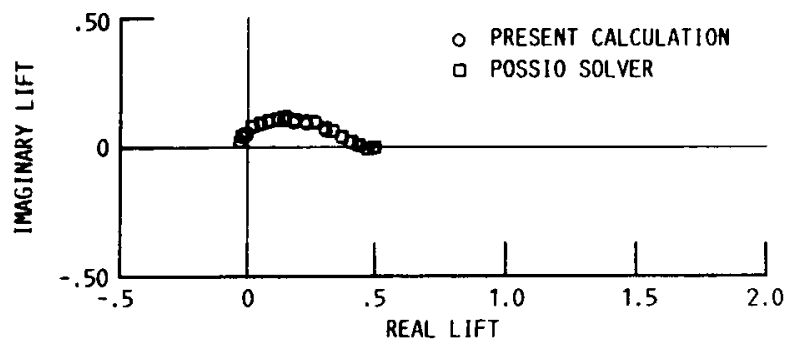

FIGURE 13. - COMPARISON BETWEEN THE COMPUTED UNSTEAOY RESPONSE FUNCTION OF A FLAT PLATE AIRFOIL IN A THREE-DIMENSIONAL GUST AT $M=0.5$ AND A POSSIO SOLVER. $k_{3}=0.442 \cdot|\vec{a}|=1, \frac{a_{2}}{a_{1}}=-\frac{7}{4}$ $k_{1}=k_{2}, \vec{a} \cdot \vec{k}=0, a_{2}>0$.

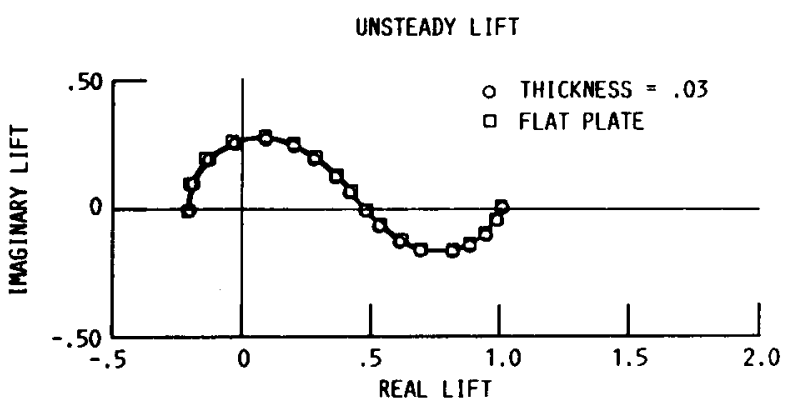

FIGURE 15. - COMPARISON BETWEEN THE COMPUTED UNSTEADY RESPONSE FUNCTION OF A FLAT PLATE AIRFOIL AND A 3 PERCENT THICK JOUKOWSKI AIRFOIL IN A ONE-DIMENSIONAL GUST AT $M=0.1$. 
UNSTEADY LIFT

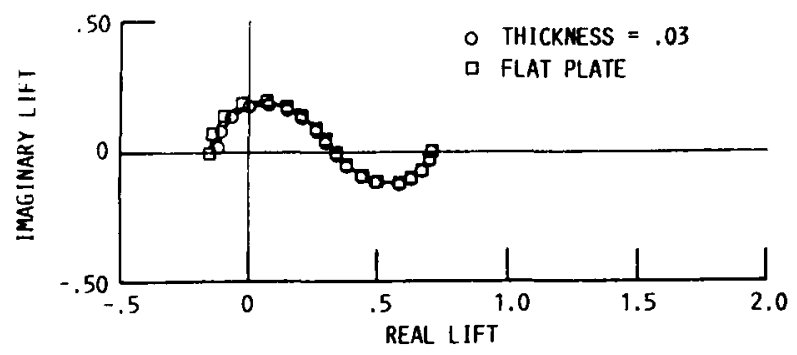

FIGURE 16. - COMPARISON BETWEEN THE COMPUTED UNSTEADY RESPONSE FUNCTION OF A FLAT PLATE AIRFOIL AND A 3 PERCENT THICK JOUKOWSKI AIRFOIL IN A TWO-DINENSIONAL GUST AT $M=0.1, \quad-a_{1}=a_{2}=.7071, k_{1}=k_{2}, a_{3}=$ $k_{3}=0$.

UNSTEADY LIFT

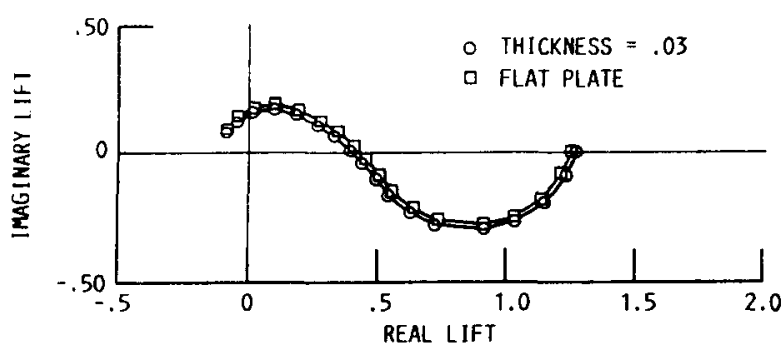

FIGURE 18. - COMPARISON BETWEEN COMPUTED UNSTEADY RESPONSE FUNCTION OF A FLAT PLATE AIRFOIL AND A 3 PERCENT THICK JOUKOWSKI AIRFOIL IN A ONE-DIMENSIONAL GUST AT $M=0.6$.
UNSTEADY LIFT

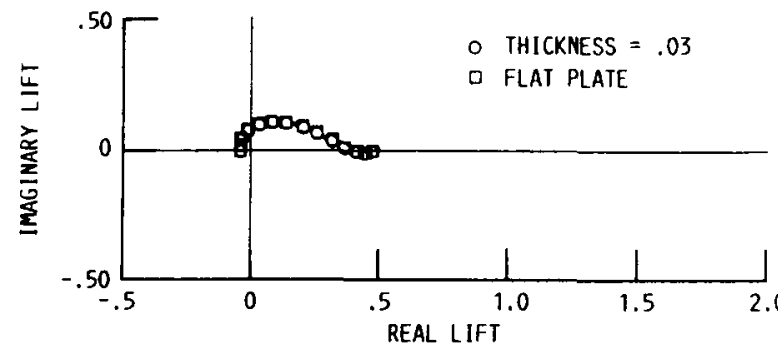

FIGURE 17. - COMPARISON BETWEEN COMPUTED UNSTEADY RESPONSE FUNCTION OF A FLAT PLATE AIRFOIL AND A 3 PERCENT THICK JOUKOWSKI AIRFOIL IN A THREEDIMENSIONAL GUST AT $M=0.1 . \quad k_{3}=0.4,|\vec{a}|=1$. $\frac{a_{2}}{a_{1}}=-\frac{7}{4} \cdot k_{1}=k_{2}, \vec{a} \cdot \vec{k}=0, a_{2}>0$.

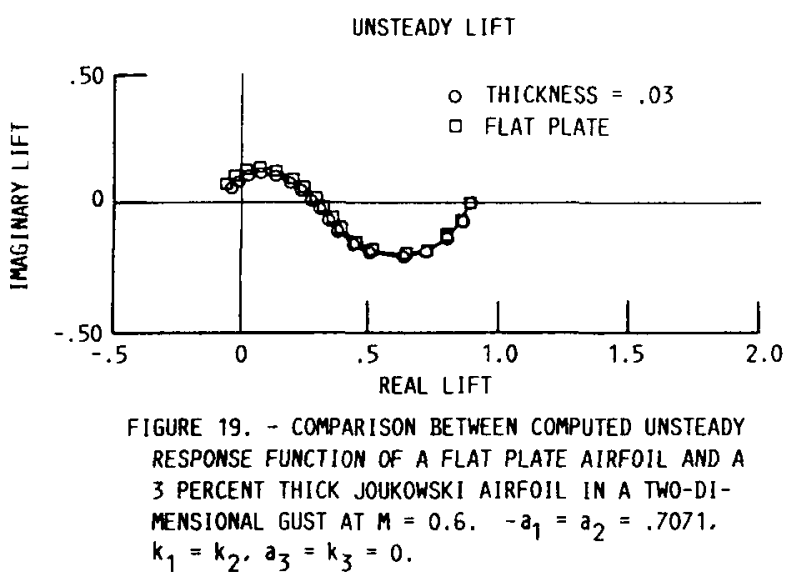

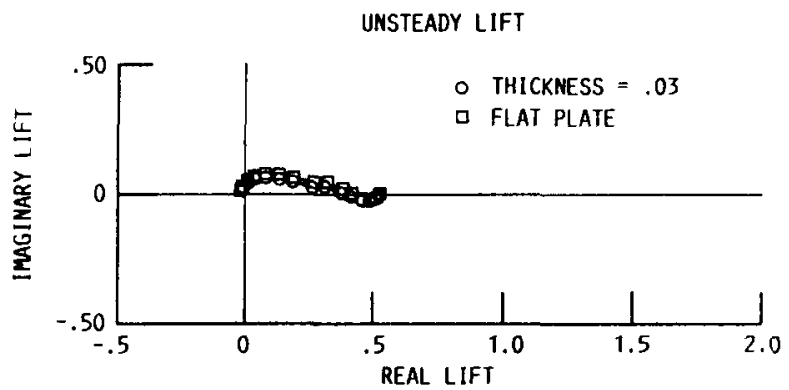

FIGURE 20. - COMPARISON BETWEEN COMPUTED UNSTEADY RESPONSE FUNCTION OF A FLAT PLATE AIRFOIL AND A 3 PERCENT THICK JOUKOWSKI AIRFOIL IN A THREE-DIMENSIOMAL GUST AT $\boldsymbol{M}=0.6, k_{3}=0.4,|\vec{a}|=1$, $\frac{a_{2}}{a_{1}}=-\frac{7}{4}, k_{1}=k_{2} \cdot \vec{a} \cdot \vec{k}=0, a_{2}>0$. 


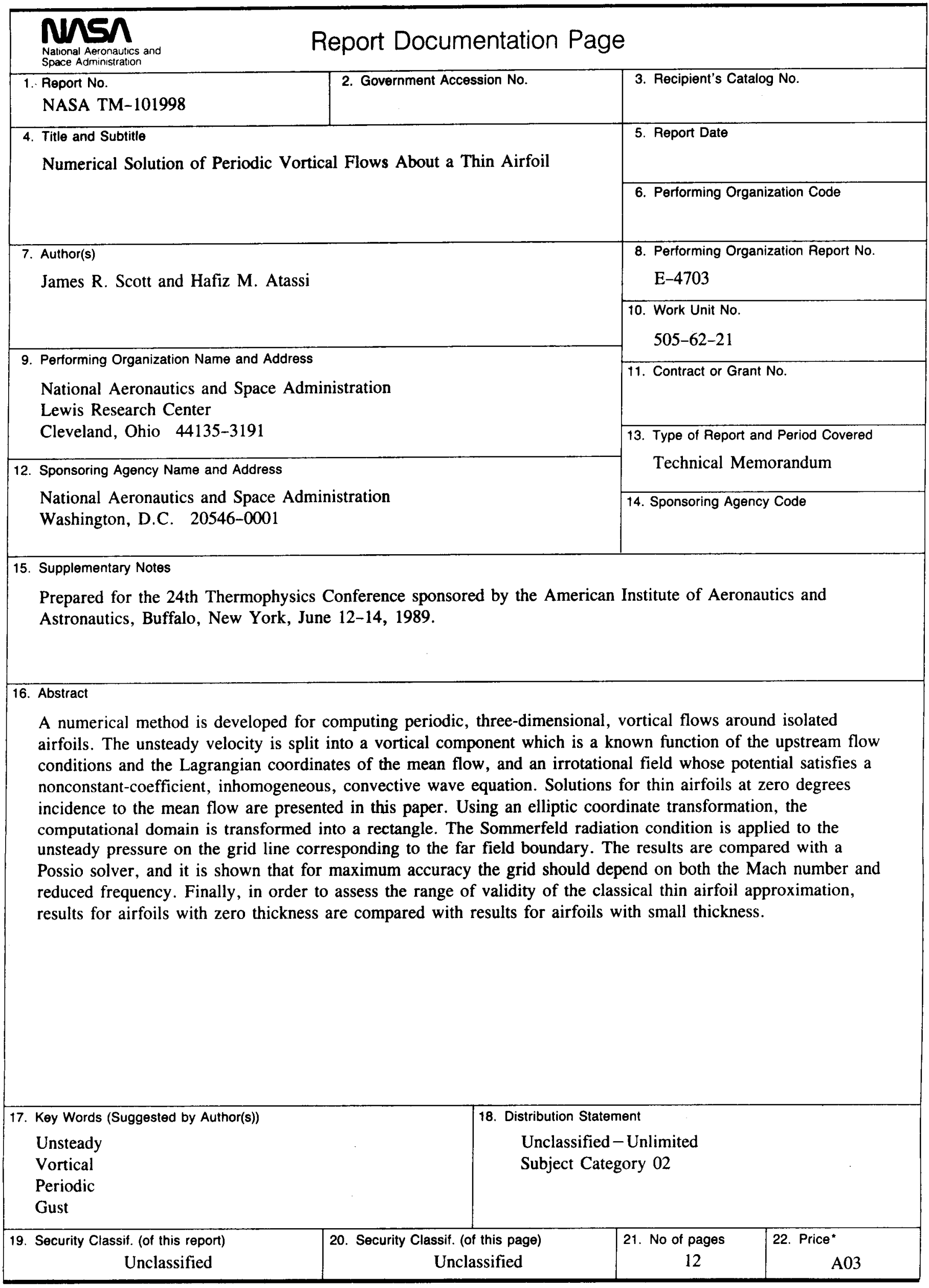

